\title{
ANÁLISIS DE LA AGROINDUSTRIA CHILENA DEL AGUACATE (PALTA) EN EL MERCADO INTERNACIONAL
}

\section{ANALYSIS OF THE CHILEAN AVOCADO (PALTA) AGROINDUSTRY IN THE THE INTERNATIONAL MARKET}

\author{
Willmer Guevara ${ }^{1 *}$, Carmen Hidalgo-Alcázar ${ }^{2}$ y Jorge L. Rojas ${ }^{3}$ \\ ${ }^{1}$ Escuela de Ciencias Empresariales, Universidad Católica del Norte, Larrondo 1281, Coquimbo, Chile. \\ ${ }^{2}$ Facultad de Ciencias Económicas y Empresariales, Universidad de León, Campus de Vegazana, 24071, \\ León, España. \\ ${ }_{3}^{3}$ Área de Procesos Industriales, Universidad Tecnológica de Chile, INACAP, Fco. de Aguirre 389, La \\ Serena, Chile. \\ *Autor para correspondencia E-mail: willmerg80@gmail.com
}

\section{RESUMEN}

El precio del aguacate (palta) (Persea americana) en el mercado interno chileno ha experimentado constantes alzas en los últimos años, siendo Chile uno de los principales productores y exportadores de este fruto a nivel mundial. El objetivo de este estudio fue evaluar el desempeño de la agroindustria del aguacate chileno en el período 2008-2017. La evaluación se llevó a cabo mediante indicadores cuantitativos de comercio, como son la metodología de Ventajas Comparativas Reveladas (VCR), la Tasa de Penetración de las Importaciones (TPI) y el Análisis de la Participación Constante de Mercado (CMS). Los análisis realizados muestran que hay una disminución de la competitividad del aguacate chileno en el mercado estadounidense, explicado por mayores volúmenes de exportaciones hacia otros mercados. Por otro lado, se observa una pérdida de ventajas comparativas debido a una baja en la producción nacional y un crecimiento de la cantidad exportada, con un leve incremento del precio pero por debajo de la media mundial. Se puede concluir que los beneficios a los exportadores, como la recuperación del IVA, el incremento de la tasa de cambio de dólar, sumados a la dinámica del comercio mundial, constituyen incentivos al incremento de las exportaciones de aguacate, lo cual impacta en los precios del mercado nacional.

Palabras clave: aguacate, Persea americana, mercado, precios, Participación Constante de Mercado, Ventajas Comparativas Reveladas, Tasa de Penetración de las Importaciones.

\section{ABSTRACT}

The price of avocado (palta) in the Chilean domestic market has steadily increased in recent years, while Chile is one the world's major producers and exporters of this fruit. The objective of this study was to evaluate the performance of the Chilean avocado agribusiness in the period 2008-2017. The evaluation was carried out using quantitative trade indicators, such as the methodology of Revealed Comparative Advantages (RCA), the Import Penetration Rate (IPR), and the Constant Market Share (CMS) analysis. The analyses conducted show that there is a decrease in the competitiveness of the Chilean avocado in the U.S. market, explained by higher exports to other markets. On the other 
hand, Chile has lost comparative advantages derived from a decrease in the domestic production of the fruit and an increase in the volumes exported, with a slight increase in the price but below the international average price. It can be concluded that the benefits to exporters such as the recovery of the VAT, the increase in the dollar exchange rate, along with the dynamics of world trade have increased exports of this fruit, causing an impact on the price of avocado in the local market.

Key words: avocado, trade, competitiveness, prices, Constant Market Share Analysis, Revealed Comparative Advantages, Import Penetration Rate.

\section{INTRODUCCIÓN}

Persea americana, comúnmente conocida como palta, aguacate o avocado, es una fruta cultivada hace siglos en América Central, donde tiene su origen, y posteriormente en América del Sur (Perú y Chile), donde se ha masificado rápidamente su consumo y comercio internacional. Estadísticamente, México es reconocido por ser el mayor productor, consumidor y exportador de aguacate (Peña et al., 2015). En cuanto a importación, tradicionalmente Estados Unidos (EE. UU.) se ha ubicado en el primer lugar, seguido por los países europeos que se proveen principalmente de aguacate mexicano, chileno y peruano (Arias et al., 2018).

Chile ha desarrollado un marco institucional capaz de apoyar al sector de exportación en el cumplimiento de las exigencias crecientes impuestas por los países de destino, lo que le ha permitido incrementar y diversificar sus exportaciones (Gutiérrez y Ferrantino, 2018). Sus exportaciones agrícolas se han enfocado tradicionalmente hacia Estados Unidos, aunque ha ido evolucionando hacia otros mercados, como el chino (Boza et al., 2020). En el caso del aguacate chileno, además de Estados Unidos, también existen acuerdos con la Unión Europea, mercado que es muy competitivo para los exportadores, por lo tanto, exige una mayor calidad y atributos organolépticos específicos para el fruto, características con las que cuenta el producto chileno, lo que le ha permitido su ingreso en este bloque (Donetti y Terry, 2014; Ferreyra et al., 2016).

La producción de aguacate en Chile ha tenido una gran expansión, debido a factores que favorecen su cultivo, como son el clima, fácil mantención de los paltos en relación con otros cultivos, la creciente demanda nacional e internacional, y excelentes resultados económicos. La superficie comercial plantada con aguacates en el año 2017 alcanzó 29.289 hectáreas, concentrándose el $95 \%$ de la superficie entre las regiones de Coquimbo y Metropolitana, fundamentalmente en Valparaíso. Este cultivo desplazó otras actividades productivas, y en la actualidad ha generado algunos conflictos sociales asociados al uso del agua, como es el caso de la provincia de Petorca (García et al., 2017; Budds, 2017; Panez-Pinto et al., 2018). Se estima que la superficie nacional está disminuyendo a una tasa promedio de crecimiento de $-1,8 \%$ para los últimos 10 años, pasando del máximo obtenido en 2007 con poco más de 35 mil hectáreas, a poco más de 29 mil hectáreas el año 2017 (Muñoz, 2018).

En Chile, el aguacate ha experimentado un alza en su precio durante los últimos años, alcanzando los \$ 5.000 pesos chilenos (CLP) por $\mathrm{kg}(1 \mathrm{US} \$=681$ CLP) en algunos sectores del país durante el año 2018. Esta situación pone en alerta a los poderes económicos del Estado, ya que la familia chilena se ve obligada a disminuir el consumo de esta fruta, la que había adquirido un papel fundamental en la dieta de cada individuo, no sólo por su sabor, sino también por sus propiedades alimenticias y aporte nutricional. Las causas atribuibles al elevado precio del aguacate aún no son claras, sin embargo, de lo que se tiene certeza, es de la influencia cada vez mayor de esta fruta en los estimadores de la Canasta Básica de Alimentos (CBA). La actualización de la CBA va de la mano con la variación del Índice de Precios al Consumidor (IPC), el cual representa la variación del costo de vida de los chilenos, y también permite estimar la evolución de la inflación, entre otros valores económicos de vital importancia (salario, costo de vida, valor de arriendos habitacionales, valor de la Unidad de Fomento (UF) y la Unidad Tributaria Mensual (UTM)).

Investigar las causas e incidencias del elevado precio del aguacate, es un tema de gran importancia para lograr formular políticas que mantengan un equilibrio entre la oferta y la demanda, y con ello evitar el incremento del costo de vida de la familia chilena. Debido a la gran apertura comercial de Chile, no solo la producción tiene efecto sobre la oferta, ya que elementos como exportaciones e importaciones son determinantes a la hora de evaluar el equilibrio oferta-demanda y el precio resultante.

En este contexto, esta investigación tiene como objetivo evaluar el desempeño de la agroindustria del aguacate chileno en el periodo 
2008 al 2017. Su evaluación se llevará a cabo mediante la representación de los principales flujos del comercio mundial de este fruto y del análisis eficiencia exportadora basada en la descomposición del valor en precio y volumen. Además, se aplicarán indicadores cuantitativos de comercio, como son la metodología de Ventajas Comparativas Reveladas (VCR), la Tasa de Penetración de las Importaciones (TPI) y el análisis de la Participación Constante de Mercado (CMS).

\section{MATERIALES Y MÉTODOS}

\section{Fuentes de información}

Los datos utilizados fueron obtenidos de la base de datos online de las Naciones Unidas (UN COMTRADE, 2020). Las series de exportaciones e importaciones están expresadas en miles de dólares corrientes y corresponden al comercio de la categoría "Aguacate (paltas), frescas o secas" código arancelario 080440.

Los datos correspondientes a la producción de aguacate fueron tomados de la base de datos de la FAOSTAT (2020). Ésta proporciona datos sobre la alimentación y la agricultura de más de 245 países, territorios y grupos regionales de la FAO.

El estudio abarca el periodo desde el año 2008 al 2017. Se escogió el 2017 como año final porque algunos de los indicadores que se utilizan dependen de los valores del periodo inicial y del periodo final, especialmente en el método CMS esto se conoce como problema de número de índice (Richardson, 1971). Chile evidencia un crecimiento sostenido de las exportaciones de aguacate hasta alcanzar un máximo en el año 2017, posterior a esa fecha se observa un quiebre. Por otra parte, el mercado de Estados Unidos fue seleccionado para el análisis de competitividad por ser el mayor importador de este bien en el mundo, y ser el país donde tradicionalmente Chile exporta su mayor cantidad de aguacate.

\section{Métodos}

Primeramente, se realizó un análisis cuantitativo de la relación entre los principales exportadores e importadores. Para su visualización se empleó un gráfico de malla, herramienta que permite presentar grandes flujos de información de manera simplificada mediante el software UCINET (Yu y Ma, 2020). Además, se realizó una descomposición de los factores de crecimiento de las exportaciones, precio, cantidad, y valor exportado utilizando la siguiente ecuación:

$$
G R_{v j}=\left[\left(X_{(k+n) j} / X_{k j}\right)^{\left(\frac{1}{n}\right)}-1\right]
$$

donde $\mathrm{GR}_{\mathrm{v}}$ : Tasa de crecimiento de la variable analizada por el país j; n: Cantidad de periodo analizado; $X_{\mathrm{kj}}$ : Valor de la variable (precio, cantidad o valor) en el periodo $\mathrm{k}$ (inicial) por el país $\mathrm{j} ; \mathrm{X}_{(\mathrm{k}+\mathrm{ni})}$ : Valor de la variable (precio, cantidad $\mathrm{o}$ valor) en el periodo $\mathrm{k}+\mathrm{n}$ (presente) por el país $\mathrm{j}$. Para profundizar en el desempeño de la agroindustria del aguacate se analizaron los siguientes indicadores:

\section{Indicador de las Ventajas Comparativas Reveladas (VCR) \\ El VCR, también conocido como índice de} Balassa, es uno de los indicadores más utilizados para estudiar los patrones del comercio internacional (Varian, 2019; Senyshyn et al., 2019; Verter et al., 2020). Este indicador permite diferenciar a los países que presentan ventaja competitiva en un producto en particular con relación a aquellos que no la tienen. Además, posibilita la comparación de las tendencias de la competitividad revelada entre los países que compiten en el mercado de esa categoría. Se utilizó este indicador con el objetivo de estudiar la competitividad entre Chile y los principales exportadores de aguacate, utilizando la siguiente expresión:

$$
V C R_{i j}=\frac{\left(X_{i j} / X_{j}\right)}{\left(X_{i} / X\right)} * 100
$$

donde:

VCR $_{\mathrm{ij}}$ : VCR del producto $\mathrm{i}$ del país $\mathrm{j} ; \mathrm{X}_{\mathrm{ij}}$ : Exportaciones de producto i desde el país $j$; $X_{j}$ : Exportaciones totales del país $\mathrm{j} ; \mathrm{X}_{\mathrm{i}}$ : Exportaciones del producto i por el mundo; $\mathrm{X}$ : Exportaciones totales del mundo.

Si el resultado del VCR es mayor a 100 indica que el país presenta una ventaja comparativa revelada (Guevara y Morales, 2018a).

\section{Tasa de Penetración de las Importaciones (TPI)}

La Tasa de Penetración de las Importaciones mide la proporción en que las importaciones abastecen la demanda interna, ya sea de un bien, sector o del conjunto de la economía nacional, suponiendo que todos los bienes importados se venden en el mercado interno. Se define como la relación entre las importaciones de un producto para un país y el consumo aparente, y éste a su vez depende de la producción reportada, las exportaciones y las importaciones (Omaña et al., 2014; Fronczek, 2017). Algebraicamente se describe como:

$$
T P I_{i j}=\frac{M_{i j}}{C_{i j}} * 100=\frac{M_{i j}}{P R_{j}-X_{i j}+M_{i j}} * 100
$$

donde:

TPI : Tasa de Penetración de las Importaciones del producto $i$ en el país $j ; \mathrm{M}_{\mathrm{ij} \text { : }}$ Importaciones del 
producto $i$ realizadas por el país $j ; \mathrm{C}_{\mathrm{ij}}$ : Consumo aparente del producto $i$ en el país $j ; \mathrm{PR}_{\mathrm{ij}}$ : Producción reportada del producto i por el país $\mathrm{j} ; \mathrm{X}_{\mathrm{ij}}$ : Exportaciones de producto i desde el país $\mathrm{j}$.

\section{Metodología de análisis de la Participación Constante de Mercado (Constant Market Share, CMS)}

El análisis de las cuotas de Participación Constante de Mercado (CMS) tiene su origen en el año 1950. Desde entonces se han planteado diferentes versiones y aplicaciones de este indicador. Entre las más relevantes se encuentran los estudios de Ahmadi-Esfahani, en los años 1995 y 2006. Otros estudios más recientes han aplicado este método en diferentes sectores industriales y países (Liu et al., 2020; Dai et al., 2020). Para este caso de estudio se empleará la variación usada por Guevara y Morales (2018b) que parte de la ecuación clásica de la participación de mercado:

$$
S_{i j k}=\frac{q_{i j k}}{Q_{i k}}
$$

donde: $S_{i j k}$ : Participación de mercado para país $j$ en el país k para el producto $\mathrm{i} ; \mathrm{q}_{\mathrm{ijk}}$ : Exportaciones del bien i, desde el país j, hacia el país o región considerado; $Q_{i k}$ : Valor de exportaciones del bien i del grupo de países competidores (el estándar) que exportan al país $\mathrm{k}$, incluye las exportaciones del país j analizado. Cuando no se utiliza el índice $\mathrm{k}$ se refiere a los parámetros mundiales.

Despejando la q y diferenciando con respecto al tiempo, se obtiene:

$$
\Delta \boldsymbol{q}_{i j k}=S_{i j k} \Delta Q_{i k}+\Delta S_{i j k} Q_{i k}
$$

La ecuación anterior sólo se mantiene durante un período de tiempo infinitamente corto. Cuando la descomposición se aplica a intervalos discretos, la ecuación puede escribirse de varias formas utilizando las variables de inicio y fin del período (Ahmadi-Esfahani, 1995), ecuación 4a, 4b y $4 \mathrm{c}$ :

$$
\begin{aligned}
\Delta q_{i j k} & =S_{i j k}^{0} \Delta Q_{i k}+\Delta S_{i j k} Q_{i k}^{1} \\
\Delta q_{i j k} & =S_{i j k}^{1} \Delta Q_{i k}+\Delta S_{i j k} Q_{i k}^{0} \\
\Delta q_{i j k} & =S_{i j k}^{0} \Delta Q_{i k}+\Delta S_{i j k} Q_{i k}^{0}+\Delta S_{i j k} \Delta Q_{i k}
\end{aligned}
$$

$\Delta$ : Variación temporal de la variable respectiva; 0 : Año inicial del análisis; 1: Año final del análisis. $\boldsymbol{S}_{\boldsymbol{i j k}}^{\mathbf{0}} \Delta \boldsymbol{Q}_{\boldsymbol{i} \boldsymbol{k}}$ : El efecto estructural que significa el cambio esperado de las exportaciones en la medida que se mantenga la participación inicial del país en el mercado mundial y en el país de destino. Si este resultado es positivo, representa que el incremento en la demanda asiste al incremento de las exportaciones (Hernández y Romero, 2009).
$\Delta \boldsymbol{S}_{\boldsymbol{i j k}} \boldsymbol{Q}_{\boldsymbol{i} \boldsymbol{k}}^{\mathbf{0}}: \quad$ El efecto competitividad mide los cambios en las exportaciones atribuido únicamente a las variaciones en las cuotas de mercado de ese país. Si el valor del coeficiente es positivo, significa que la competitividad ha aumentado (Dieter y Englert, 2006).

$\Delta \boldsymbol{S}_{\boldsymbol{i j k}} \Delta \boldsymbol{Q}_{\boldsymbol{i} \boldsymbol{k}}$ : El efecto de segundo orden, es el efecto dinámico que captura la interacción de los cambios en la cuota de mercado con los cambios en la demanda (Ahmadi-Esfahani, 1995).

Un segundo nivel de descomposición introduce otro nivel con seis efectos adicionales. En este caso particular se realiza una adecuación para un solo mercado k. y un solo bien i.

$$
\begin{aligned}
& \Delta \boldsymbol{q}_{i j k}=\boldsymbol{S}_{i j}^{\mathbf{0}} \Delta \boldsymbol{Q}_{i k}+\left(\boldsymbol{S}_{i j k}^{\mathbf{0}} \Delta \boldsymbol{Q}_{i k}-\boldsymbol{S}_{i j}^{\mathbf{0}} \Delta \boldsymbol{Q}_{i k}\right)+ \\
& \Delta \boldsymbol{S}_{i j} \boldsymbol{Q}_{i k}^{\mathbf{0}}+\left(\Delta \boldsymbol{S}_{i j k} \boldsymbol{Q}_{i k}^{\mathbf{0}}-\Delta \boldsymbol{S}_{i j} \boldsymbol{Q}_{i k}^{\mathbf{0}}\right)+ \\
& \left(\frac{Q_{i}^{\mathbf{1}}}{\boldsymbol{Q}_{i}^{\mathbf{0}}}-1\right)\left(\Delta \boldsymbol{S}_{i j k} \boldsymbol{Q}_{i k}^{\mathbf{0}}\right)+\left(\Delta \boldsymbol{S}_{i j k} \Delta \boldsymbol{Q}_{i k}-\right. \\
& \left(\left(\frac{Q_{i}^{1}}{Q_{i}^{\mathbf{0}}}-1\right)\left(\Delta \boldsymbol{S}_{i j k} \boldsymbol{Q}_{i k}^{\mathbf{0}}\right)\right)
\end{aligned}
$$

$\boldsymbol{S}_{\boldsymbol{i j k}}^{\mathbf{0}} \Delta \boldsymbol{Q}_{\boldsymbol{i k}}$ : El efecto crecimiento muestra el incremento en las exportaciones como consecuencia de un aumento en la demanda, mientras la participación del país exportador en el mercado mundial permanece constante. El signo positivo de éste, indica que el país se ha visto favorecido por el crecimiento de la demanda mundial (Singh y Mohan, 2011).

$\left(\boldsymbol{S}_{\boldsymbol{i j k}}^{\mathbf{0}} \Delta \boldsymbol{Q}_{\boldsymbol{i j k}}-\boldsymbol{S}_{\boldsymbol{i j}}^{\mathbf{0}} \Delta \boldsymbol{Q}_{\boldsymbol{i} \boldsymbol{k}}\right)$ : El efecto mercado es el cambio en las exportaciones, siempre y cuando el exportador mantenga constante su participación en el mercado de destino y mundial. Si el signo es positivo implica una concentración de las exportaciones en el mercado analizado (AhmadiEsfahani, 2006).

$\Delta \boldsymbol{S}_{\boldsymbol{i j}} \boldsymbol{Q}_{\boldsymbol{i} \boldsymbol{k}}^{\mathbf{0}}$ : El efecto residual puro o efecto competitividad general evalúa el cambio en las exportaciones que se espera que ocurra como consecuencia de un cambio en la competitividad general (Ahmadi-Esfahani, 1995).

$\left(\Delta \boldsymbol{S}_{\boldsymbol{i j k} \boldsymbol{k}} \boldsymbol{Q}_{\boldsymbol{i} \boldsymbol{k}}^{\mathbf{0}}-\Delta \boldsymbol{S}_{\boldsymbol{i} \boldsymbol{j}} \boldsymbol{Q}_{\boldsymbol{i} \boldsymbol{i}}^{\mathbf{0}}\right): \quad$ El efecto residual estructural estático es el cambio en las exportaciones del bien, motivado por un cambio en la competitividad en un mercado meta ( $k$ ) (Ahmadi-Esfahani, 1995; Torres, 2009).

$\left(\frac{\boldsymbol{Q}_{i}^{\mathbf{i}}}{\boldsymbol{Q}_{\boldsymbol{i}}^{\mathbf{0}}}-1\right)\left(\Delta \boldsymbol{S}_{\boldsymbol{i j k} \boldsymbol{k}} \boldsymbol{Q}_{\boldsymbol{i} \boldsymbol{k}}^{\mathbf{0}}\right):$ El efecto de segundo orden puro mide la interacción entre el cambio en la participación del exportador en el mercado meta (k), y el cambio en la demanda mundial (Hernández y Romero, 2009).

$$
\left(\Delta \boldsymbol{S}_{i j k} \Delta \boldsymbol{Q}_{i k}-\left(\left(\frac{\boldsymbol{Q}_{i}^{\mathbf{1}}}{\boldsymbol{Q}_{\boldsymbol{i}}^{\mathbf{0}}}-1\right)\left(\Delta \boldsymbol{S}_{\boldsymbol{i j k} \boldsymbol{k}} \boldsymbol{Q}_{\boldsymbol{i} \boldsymbol{k}}^{\mathbf{0}}\right)\right):\right. \text { El efecto }
$$


residual estructural dinámico determina la interacción del porcentaje de mercado del país de enfoque con los cambios en la estructura de la demanda mundial. Cuando es positivo, la demanda mundial está creciendo rápidamente para aquellos productos cuya participación en las exportaciones del país de enfoque está aumentando (Guevara y Morales, 2018b).

\section{RESULTADOS Y DISCUSIÓN}

\section{El mercado mundial del aguacate}

Chile alcanzó su mayor valor de exportación de palta en el año 2017, registrando a nivel mundial un valor de 5.823,3 millones de dólares. La mayor participación de mercado la tuvo México con un 49,8\%; le siguen Países Bajos con un 10,9\%; Perú con un $10 \%$; Chile con $8,23 \%$; España y otros 5 países que concentran el $7,8 \%$ de las exportaciones. Estos vendedores se relacionaron fundamentalmente con 10 países compradores (Fig. 1). Los importadores fueron liderados por
EE. UU. con un $44,7 \%$, abasteciéndose en un 88,3\% de México, y el resto de Perú y Chile. Países Bajos como segundo importador, es el país más activo en el comercio de aguacate, en su rol de reexportado. En los años 2018 y 2019 Chile vio caer sus exportaciones significativamente en un contexto donde las exportaciones mundiales de este fruto estuvieron al alza.

\section{Eficiencia exportadora precio, volumen y valor}

Para evaluar la eficiencia exportadora se considera el crecimiento de las variables precio, cantidad y valor de las exportaciones de aguacate de los países analizados respecto a la media mundial de cada variable. El crecimiento del valor es la resultante del efecto precio y cantidad, sin embargo, para que el crecimiento de las exportaciones sea eficiente en el país, debería tener una tasa de crecimiento de estas dos variables sobre la media del valor mundial. En el caso del precio, no debería ser menor a la inflación interna del país analizado, porque implicaría que

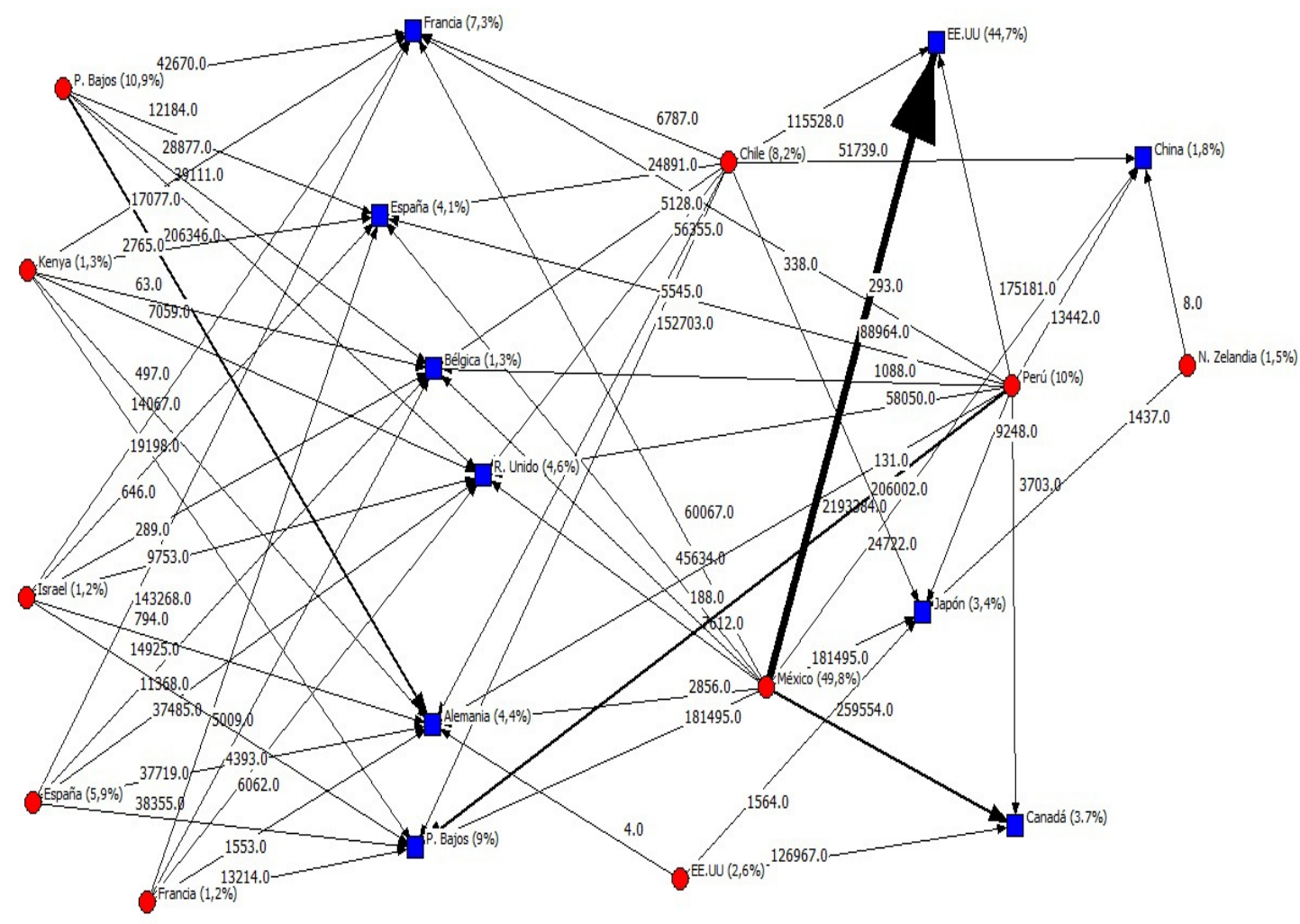

Exportador.

Importador.

Fig. 1. Relación entre principales exportadores (Top 10) e importadores (Top 10) de aguacate, año 2017 (miles US\$). 1 US $\$=681$ CLP

Fig. 1. Relationship between the main avocado exporters (Top 10) and importers (Top 10), year 2017 (thousand US\$).1 US\$ = 681 CLP

Fuente: Elaborado con información de UN COMTRADE, 2020. 
una proporción de los costos están creciendo más que los ingresos externos.

En la Fig. 2 se puede observar que para Chile el precio creció solo un $1 \%$ promedio anual, el más bajo de los países analizados, muy por debajo de $4,1 \%$ mundial, y de la tasa de inflación nacional que supera el 3\% anual en la última década, según información del Instituto Nacional de Estadísticas (INE) (Galará et al., 2018). Su tasa de crecimiento en cantidad exportada fue de $10,7 \%$, por debajo de 11,3\% mundial, la combinación de ambas variables significó un crecimiento del valor de la tasa a $11,8 \%$ en el periodo $2008-2017$, inferior al 15,9\% mundial. Los países de mejor crecimiento en el precio fueron EE. UU. con 6,3\%, Nueva Zelandia con 5,7\%, Israel con 5,65\%, y México con 4,3\%.

\section{Resultados de los indicadores.}

Ventajas Comparativas Reveladas (VCR)

De acuerdo con los resultados indicados en la Fig. 3, EE. UU. no presenta ventaja comparativa del producto "aguacate", pues para el año 2017 registró un VCR de 30, similar a los años anteriores. Esta situación se explica porque si bien este país presenta un alto nivel de producción, el aguacate no tiene un aporte relevante, debido a que la producción de aguacate la destinan mayormente al consumo interno. Los cinco países restantes que fueron estudiados con fines comparativos mostraron tener una elevada ventaja comparativa.

Perú lidera este indicador, evidenciando un incremento durante los diez años analizados, superando a países tradicionales como México y Chile. Chile muestra un descenso sostenido desde el año 2009, llegando a su valor más bajo en el año 2015. Si bien su ventaja comparativa va en descenso, no se podría desestimar su aporte a la cuota mundial del mercado del aguacate como exportador directo.

\section{Tasa de Penetración de las Importaciones (TPI)}

La competitividad en los mercados internos de los países estudiados está liderada por México, Perú, Israel, Kenia y Nueva Zelandia, con un TPI de $0 \%$, es decir, los productores de esos países logran satisfacer la totalidad de la demanda interna (Guevara y Morales, 2018b). En Chile, la tendencia del indicador ha venido al alza desde el año 2015, lo que indica su mayor dependencia de las importaciones para abastecer el mercado interno (Tabla 1).

España, EE. UU., Francia y Países Bajos, presentan un elevado índice de penetración interno, con una media que está sobre el $79 \%$, lo que indica que estas naciones son incapaces de abastecer su demanda interna y por ello requieren de producto extranjero. En el caso de los Países Bajos han encontrado una gran oportunidad en la

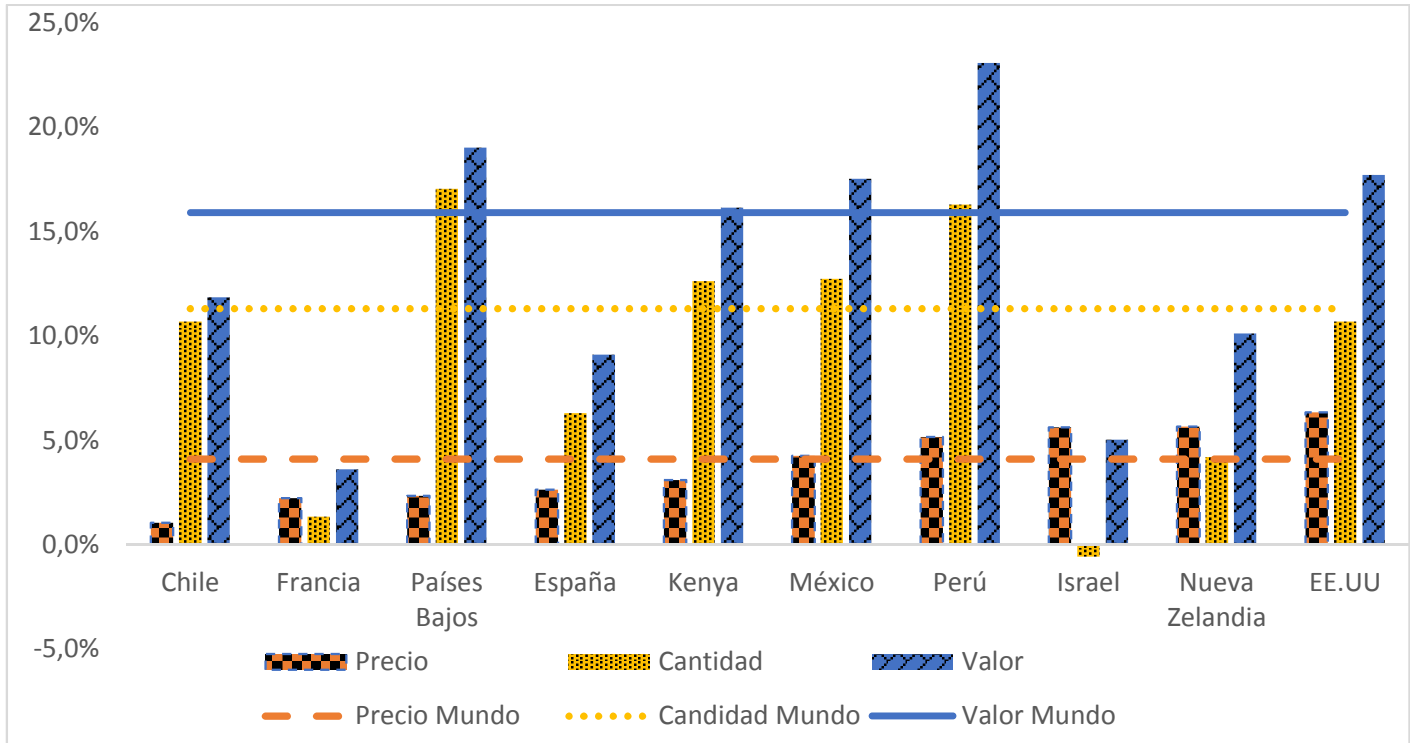

Fig. 2. Tasa de crecimiento promedio anual del precio, cantidad y valor de los principales exportadores de aguacate, 2008-2017 (\%).

Fig. 2. Average annual growth rate of price, quantity, and value of the main avocado exporters, 20082017 (\%).

Fuente: Elaboración con información de UN COMTRADE, 2020. 


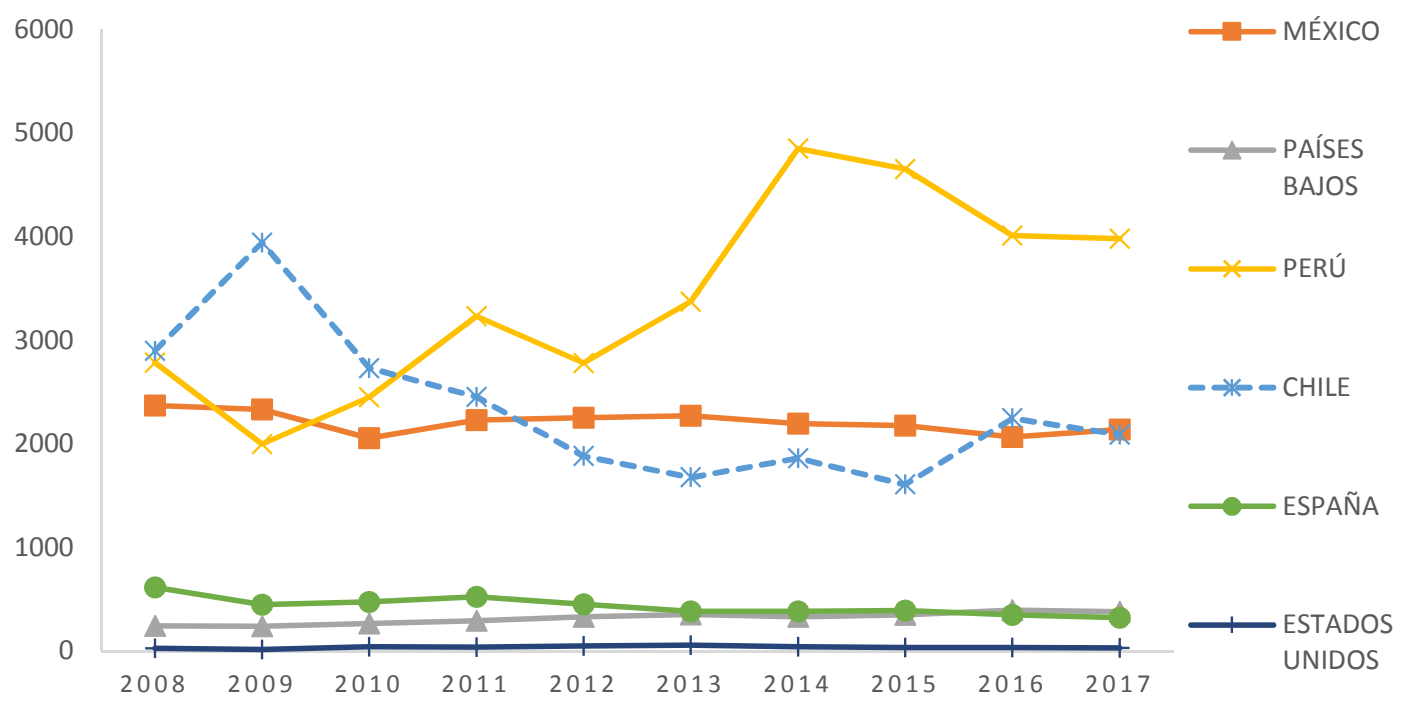

Fig. 3. Índice de Ventaja Comparativa Revelada, principales exportadores de aguacate, 2008-2017. Fig. 3. Index of Revealed Comparative Advantage, main avocado exporters, 2008-2017.

Fuente: Elaborado con información de UN COMTRADE, 2020.

Tabla 1. Tasa de Penetración de las Importaciones (\%) de los principales exportadores de aguacate, 2008-2017.

Table 1. Import Penetration Rate (\%) of the main avocado exporters, 2008-2017

\begin{tabular}{lrrrrrrrrrrr}
\hline Países & $\mathbf{2 0 0 8}$ & $\mathbf{2 0 0 9}$ & $\mathbf{2 0 1 0}$ & $\mathbf{2 0 1 1}$ & $\mathbf{2 0 1 2}$ & $\mathbf{2 0 1 3}$ & $\mathbf{2 0 1 4}$ & $\mathbf{2 0 1 5}$ & $\mathbf{2 0 1 6}$ & $\mathbf{2 0 1 7}$ & Media \\
\hline México & 0 & 0 & 1 & 0 & 0 & 0 & 0 & 0 & 0 & 0 & 0 \\
Perú & 0 & 0 & 0 & 0 & 0 & 0 & 0 & 0 & 0 & 0 & 0 \\
Chile & 1 & 0 & 0 & 2 & 1 & 5 & 5 & 14 & 243 & & 30 \\
España & 63 & 60 & 43 & 66 & 67 & 87 & 94 & 92 & 100 & 117 & 79 \\
Estados Unidos & 79 & 62 & 73 & 69 & 71 & 82 & 84 & 84 & 92 & 92 & 79 \\
Francia & 125 & 110 & 109 & 114 & 122 & 116 & 117 & 119 & 116 & 117 & 116 \\
Israel & 0 & 0 & 0 & 0 & 0 & 0 & 0 & 0 & 0 & 0 & 0 \\
Kenia & 0 & 0 & 0 & 0 & 0 & 0 & 0 & 0 & 0 & 3 & 0 \\
Nueva Zelanda & 0 & 1 & 1 & 0 & 0 & 0 & 0 & 0 & 0 & 0 & 0 \\
Países bajos & 488 & 631 & 398 & 504 & 558 & 462 & 391 & 403 & 485 & 1239 & 556 \\
\hline
\end{tabular}

Fuente: Elaboración con información de UN COMTRADE (2020) y FAOSTAT (2020).

reexportación de aguacate, dedicando gran parte de sus importaciones a la venta a países europeos.

Para Chile las exportaciones han venido en aumento desde el año 2015, mientras que la importación de aguacate ha tenido disminuciones importantes en los años 2012 y 2014. Según estadísticas de la ODEPA, el consumo de aguacate por la población chilena ha incrementado de 21 mil toneladas en el año 2007 a 31 mil en el año 2016. A partir de esto, se podría inferir la incapacidad del mercado nacional para abastecer la demanda, provocado aparentemente por el decrecimiento productivo durante los últimos tres años, lo que trae como consecuencia un precio elevado para los consumidores.

Chile, al ser un país productor de aguacate, se esfuerza por mantener un flujo de producción continuo; lamentablemente estos niveles han caído en los últimos tres años, con una producción en el año 2016 de 140.558 toneladas y una exportación de 147.124 toneladas. De esta forma, el aguacate chileno no es suficiente para abastecer la demanda nacional y es indispensable importarla desde Perú principalmente, lo que se traduce en tiempos de espera y costos asociados, conocido como el efecto retardado. 
Esta situación, en la que las exportaciones supera a la producción, se agudizó en el año 2017, lo que para el efecto del cálculo del consumo aparente resultó ser negativo, algo que en la práctica es imposible, y puede explicarse por la producción no reportada.

Un antecedente a tener en cuenta, son los beneficios para los exportadores en Chile. De acuerdo con el Decreto de Ley $\mathrm{N}^{\circ} 825$, los exportadores tienen derecho a recuperar el IVA, por lo que se hace aún más lucrativo vender el producto en el extranjero que en el mercado nacional, favorecido además, por el incremento del cambio de dólar frente al peso chileno. Estos dos factores, sumados a la dinámica del comercio mundial y a la caída en la producción en Chile, están entre las causas del aumento del precio en el mercado nacional y por consiguiente, su efecto en el consumo nacional.

\section{Participación Constante de Mercado (CMS)}

Estados Unidos concentra el $44,6 \%$ de las importaciones de aguacate a nivel mundial, y es abastecido fundamentalmente por México, Perú y Chile. Por tanto, se aplicó el método CMS en este conjunto de países, tomando como inicio el año 2014, debido a que ese fue el año en que el precio de venta de esta fruta comenzó a ir en alza constante, y el año 2017 por ser el año donde Chile alcanzó su máximo valor exportado en su historia.

Los resultados del primer nivel de descomposición (Tabla 2) muestran un aumento de las exportaciones de todos los países estudiados en el mercado norteamericano. En el caso de Perú y Chile, han mantenido una tasa de crecimiento de $13 \%$ y $16 \%$, respectivamente, para el periodo analizado, muy inferior al creciente de México que alcanzó una tasa de $25 \%$, casi duplicando el valor de sus exportaciones en el periodo.

El aumento sostenido de las importaciones de Estados Unidos, sumado a su incapacidad de satisfacer el consumo nacional, permitió beneficiar a los países exportadores. Situación evidenciada en el efecto estructural el cual tomó valores positivos para todos sus países proveedores de aguacates.

El efecto competitividad, evidencia que Chile y Perú han perdido competitividad, espacio que ha sido ganado por México. Por tanto, el efecto de segundo orden (interacción) tuvo un resultado negativo para ambos países sudamericanos.

En el segundo nivel de descomposición presentado en la Tabla 3, se evidencian 6 efectos adicionales al CMS. En primer lugar, el efecto crecimiento beneficia a todos los países analizados,

Tabla 2. CMS del aguacate en el mercado norteamericano, principales exportadores, primer nivel, años 2014-2017 (miles US\$).

Table 2. CMS of avocado in the North American market, main exporters, first level, years 2014-2017 (thousands US\$).

\begin{tabular}{lcccc}
\hline & $\begin{array}{c}\text { Cambio en las } \\
\text { exportaciones }\end{array}$ & $\begin{array}{c}\text { Efecto } \\
\text { estructural }\end{array}$ & $\begin{array}{c}\text { Efecto } \\
\text { competitividad }\end{array}$ & $\begin{array}{c}\text { Efecto segundo } \\
\text { orden }\end{array}$ \\
\hline México & $1,081,853.0$ & $998,882.0$ & $43,699.9$ & $39,271.1$ \\
Perú & $54,216.0$ & $108,705.7$ & $-28,699.1$ & $-25,790.6$ \\
Chile & $43,234.0$ & $71,715.3$ & $-15,000.8$ & $-13,480.5$
\end{tabular}

Fuente: Elaborado con información de UN COMTRADE, 2020.

$1 \mathrm{US} \$=681$ CLP

Tabla 3. CMS del aguacate en el mercado norteamericano, principales exportadores, segundo nivel, años 2014-2017 (miles US\$).

Table 3. CMS of avocado in the North American market, main exporters, second level, years 20142017 (thousands US\$).

\begin{tabular}{lcccccc}
\hline & $\begin{array}{c}\text { Efecto } \\
\text { crecimiento }\end{array}$ & $\begin{array}{c}\text { Efecto } \\
\text { mercado }\end{array}$ & $\begin{array}{c}\text { Efecto } \\
\text { residual } \\
\text { puro }\end{array}$ & $\begin{array}{c}\text { Efecto } \\
\text { residual } \\
\text { estructural } \\
\text { estático }\end{array}$ & $\begin{array}{c}\text { Efecto } \\
\text { segundo } \\
\text { orden puro }\end{array}$ & $\begin{array}{c}\text { Efecto } \\
\text { residual } \\
\text { estructural } \\
\text { dinámico }\end{array}$ \\
\hline México & $857,324.7$ & $141,557.3$ & $1,319.1$ & $42,380.8$ & $47,037.1$ & $-7,766.0$ \\
Perú & $184,405.5$ & $-75,699.8$ & $-14,074.8$ & $-14,624.3$ & $-30,890.7$ & $5,100.2$ \\
Chile & $137,572.8$ & $-65,857.5$ & $12,755.7$ & $-27,756.4$ & $-16,146.3$ & $2,665.8$ \\
\hline
\end{tabular}

Fuente: Elaborado con información de UN COMTRADE, 2020.

$1 \mathrm{US} \$=681 \mathrm{CLP}$ 
debido a que éstos son los países que aportan la mayor cuota de aguacate en el mundo. Esto, sumado al aumento de la demanda y al valor internacional del fruto, plantea un escenario beneficioso para los grandes exportadores.

Por su parte, el efecto mercado arroja valores negativos para Perú y Chile. Éstos se explican por el cambio de destino de sus exportaciones, es decir, se concentraron más en otros mercados que en el mercado estadounidense. Por su parte, México concentra la mayor parte de las exportaciones al mercado de destino analizado.

El efecto residual puro impactó negativamente en Perú, por lo que su competitividad general se vio afectada. Por el contrario, los resultados de Chile indican que mejoró su competitividad global al igual que México. Por su parte, el efecto residual estructural estático es negativo, tanto para Chile como para Perú, especialmente este último presenta ambos efectos negativos, sin embargo, su pérdida de competitividad fue mayor en el mercado americano.

La interacción entre el cambio en la participación del exportador en el mercado de EE. UU., y el cambio en la demanda mundial (efecto de segundo orden puro) mantiene una influencia negativa sobre Chile y Perú, es decir, estos países han perdido participación en EE. UU. cuando la demanda mundial de aguacate está aumentando. Finalmente, el efecto residual estructural dinámico es negativo para México, ya que sus exportaciones no aumentaron hacia otras partes del mundo por concentrarse en abastecer mayormente a EE. UU., dado por la cercanía geográfica de estos países. En contraposición, Chile y Perú mostraron una mayor tendencia de crecimiento a los mercados mundiales que al mercado meta analizado.

De acuerdo con esta metodología, Perú y Chile han perdido competitividad en el mercado estadounidense. Sin embargo, han tenido mejor desempeño en otros mercados mundiales. México sigue siendo el líder indiscutible, consolidándose en el mercado de EE. UU como el mayor proveedor de aguacate, también con buen desempeño en otros mercados globales, pero con una mayor concentración hacia el mercado estadounidense. Estos resultados evidencian un resultado negativo para Chile, pero a la vez puede significar el inicio del cambio de sus patrones comerciales agroindustriales mediante la diversificación a otros destinos. Este cambio se evidencia en el aumento de las exportaciones hacia Países Bajos, Reino Unido y Argentina. También un enfoque hacia los países asiáticos puede suponer una posibilidad para Chile, en especial en China, donde en el año 2014 se registraron exportaciones a un precio superior a los demás mercados. Este mercado puede ser potencialmente interesante por la baja concentración de exportadores de este bien y la importante transformación económica que ha beneficiado a sus ciudadanos, los cuales han aumentado significativamente su poder adquisitivo.

\section{CONCLUSIONES}

El crecimiento de las exportaciones de Chile se sustenta en el incremento de la cantidad exportada y en un leve crecimiento del precio de exportación, es decir, que, para mantener una participación de la cuota de mercado en valor, ha dependido del aumento del volumen de exportación. En tal sentido, la identificación y enfoque hacia aquellos mercados con mayor dinamismo del precio puede ayudar a mejorar la competitividad global de las exportaciones de aguacates chilenos, política que parece estar empezando a aplicarse según los resultados del CMS, que indican que está existiendo un cambio en el enfoque que tradicionalmente había sido hacia EE. UU.

De acuerdo con el concepto de VCR se determinó que Chile está perdiendo eficiencia en su producción de aguacate. Antes del año 2010, se mostraba como el país líder en este indicador y en la actualidad ocupa el tercer puesto, siendo superado por Perú y México. En la práctica dadas las condiciones productivas de Chile, especialmente teniendo en cuenta la situación hídrica existente en las zonas de cultivo, se ve muy difícil competir en valor exportado con estos países. Sin embargo, desde el punto de vista comercial, se puede desarrollar la estrategia de importar productos de aquellos países con menos reputación en el mercado internacional, comprando producto a menor precio para la distribución en el mercado nacional, y exportando parte de la producción nacional a aquellos países que valoran positivamente los atributos del aguacate chileno, similar al modelo desarrollado por los Países Bajos.

La TPI en Chile ha experimentado un aumento importante desde el año 2013. En el año 2016, ya toda la demanda interna dependía de las importaciones, explicado esto por el gran crecimiento de las exportaciones y la disminución en la producción informada. Teniendo en cuenta la tradición de consumo de aguacate en Chile, sería importante diseñar una estrategia que permita potenciar su cultivo a través de programas de incentivo a la agricultura semiurbana, especialmente aprovechando las potencialidades que tiene el uso de aguas grises y la aplicación de técnicas innovadoras para el uso racional del agua en proyectos agroindustriales. Para lograrlo, es necesario invertir en investigación enfocada en 
este tema, promovida tanto por el gremio como por iniciativas gubernamentales.

Se espera que los resultados de este estudio contribuyan a la toma de decisiones en cuanto a la política de comercio exterior del aguacate. Por otra parte, la metodología planteada puede ser replicada con facilidad para evaluar otros productos que presentan una influencia importante en el consumo nacional, y a la vez constituyen una fuente de ingresos importante para el país mediante la exportación, como por ejemplo el limón, pescados, mariscos, entre otros.

\section{LITERATURA CITADA}

Ahmadi-Esfahani, F.Z. 1995. Wheat market shares in the presence of Japanese import quotas. Journal of Policy Modeling 17:315-323. doi. org/10.1016/0161-8938(94)00036-F.

Ahmadi-Esfahani, F.Z. 2006. Constant market shares analysis: uses, limitations and prospects. The Australian Journal of Agricultural and Resource Economics 50:510-526. doi.org/10.1111/j.14678489.2006.00364.x.

Arias, F., C. Montoya, y O. Velásquez. 2018. Dinámica del mercado mundial de aguacate. Revista Virtual Universidad Católica del Norte (55):30-48. doi:10.35575/rvucn.n55a2

Boza, S., J. Muñoz, A. Núñez, y J. Díaz-Lanchas. 2020. Dinámica de las exportaciones frutícolas chilenas desde una perspectiva regional (2008-2018). Chilean Journal of Agricultural \& Animal Sciences, ex AgroCiencia 36(1):26-34.

Budds, J. 2017. Acceso a los recursos de agua para la agricultura en el valle de La Ligua, Chile. Revista Derecho Administrativo Económico 11:371-379. doi.org/10.7764/redae.11.10.

Dai, Y.Y., Y. Yuan, Y. Yuan, Z. Zhou, and H.Y. Zhang. 2020. Competitiveness of Chinese and Indonesian tilapia exports in the US market. Aquaculture International 28(2):791804.

Dieter, M., and H. Englert. 2006. Competitiveness in the global forest industry sector: an empirical study with special emphasis on Germany. European Journal of Forest Research 126(11):401-412. doi.org/10.1007/ s10342-006-0159-x.

Donetti, M., and L.A. Terry. 2014. Biochemical markers defining growing area and ripening stage of imported avocado fruit cv. Hass. Journal of Food Composition and Analysis 34:90-98. doi.org/10.1016/j.jfca.2013.11.011.

FAOSTAT. 2020. Bases de datos de producción. Disponible en http://www.fao.org/faostat/ es/\#data/QC (Consulta 15 marzo 2020).
Ferreyra, R., G. Sellés, J. Saavedra, J. Ortiz, C. Zúñiga, C. Troncoso, et al. 2016. Identification of pre-harvest factors that affect fatty acid profiles of avocado fruit (Persea americana Mill) cv. 'Hass' at harvest. South African Journal of Botany 104:15-20. doi.org/10.1016/j. sajb.2015.10.006

Fronczek, M. 2017. Import penetration rate in view of a new concept of measuring foreign trade. Argumenta Oeconomica 1(38):285-297.

Galará, I. A., P. Hueichapan, R. Lineros, y M. Montes. 2018. Análisis de volatilidad y descomposición de variabilidad del IPC en Chile. Instituto Nacional de Estadísticas (INE), Santiago, Chile.

García, P.B., F.H. Olguín, C.C. Mahn, y A.S. Cuevas. 2017. La eco-geo-política del agua: una propuesta desde los territorios en las luchas por la recuperación del agua en la provincia de Petorca (Zona central de Chile). Revista Rupturas 8(1):167-199. doi. org/10.22458/rr.v8i1.1977.

Guevara W., y C Morales. 2018a. Análisis de la competitividad exportadora de los principales productos exportados por Chile y Perú. GCG: Revista de Globalización, Competitividad y Gobernabilidad 12(2):3856. doi.org/10.3232/GCG.2018.V12.N2.02.

Guevara W., and C. Morales. 2018b. Performance of the Chilean wine industry through trade indicators in the period 2001-2016. Bulgarian Journal of Agricultural Science 24(3):341-351.

Gutiérrez, S.A., and M.J. Ferrantino. 2018. Export diversification and structural dynamics in the growth process: The case of Chile. p. 51-73. In Export Dynamics and Economic Growth in Latin America: A Comparative Perspective. Routledge, New York, USA.

Hernández, R.A., e I. Romero. 2009. Módulo para analizar el crecimiento del comercio internacional (MAGIC Plus) Manual para el usuario. CEPAL Serie Estudios y Perspectivas, México D.F., México.

Liu, Y., X. Shi, and J. Laurenceson. 2020. Dynamics of Australia's LNG export performance: A modified constant market shares analysis. Energy Economics 89(104808):1-8. doi.org/10.1016/j.eneco.2020.104808.

Muñoz, V. 2018. La palta chilena en los mercados internacionales. Oficina de Estudios y Políticas Agrarias (ODEPA), Santiago, Chile.

Omaña, J.M, I. Almora, B. Cruz, G. Hoyos, J. Quintero, y M. Fortis. 2014. Competitividad de la carne de ganado bovino entre los países miembros del TLCAN 1997-2008. Revista Mexicana de Ciencias Agrícolas 5(2):175-189. 
Panez-Pinto, A., P. Mansilla-Quiñones, y A. Moreira-Muñoz. 2018. Agua, tierra y fractura sociometabólica del agronegocio. Actividad frutícola en Petorca, Chile. Revista Bitácora Urbano Territorial 28(3):153-160.

Peña, L.S., S. Rebollar, N. Callejas, J. Hernández, y G. Gómez. 2015. Análisis de viabilidad económica para la producción comercial de aguacate Hass. Revista Mexicana de Agronegocios 36:1325-1338.

Richardson, D. 1971. Some Sensitivity Tests for a "Constant-Market-Shares" Analysis of Export Growth. The Review of Economics and Statistics 53 (3):300-304.

Senyshyn, O., O. Kundytskyj, and O. Klepanchuk. 2019. An index analysis for the assessment of the competitiveness of food products in Ukraine. Journal of Competitiveness 11(2):130-143. doi. org/10.7441/joc.2019.02.09.

Singh, K., and M.M. Dey. 2011. International competitiveness of catfish in the US market: A constant market share analysis. Aquaculture Economics \& Management 15(3):214-229.

Torres, V.H. 2009. La competivitidad del aguacate mexicano en el mercado estadounidense. Revista de Geografía Agrícola. Universidad Autónoma de Chapingo 43(25039):1-40.
Troncoso, J.L., M. Aguirre, P. Manriquez, and D. Mundigo. 2008. Influence of size, destination market and month of sale of Chilean avocados: A hedonic approach. Revista Ciencia e Investigación Agraria 35(3):333339.

UN Comtrade Database. 2020. Disponible en https://comtrade.un.org/data/.(Consulta 1 abril 2020).

Varian, B.D. 2019. The manufacturing comparative advantages of late-Victorian Britain. Cliometrica 1-28. doi.org/10.1007/ s11698-019-00195-w.

Verter N., I. Zdráhal, V. Bečvářová, and L. Grega. 2020. 'Products mapping' and trade in agrifood products between Nigeria and the EU28. Agric. Econ. - Czech 66:34-45. doi. org/10.17221/145/2019-AGRICECON.

Yu, J., and J Ma. 2020. Social network analysis as a tool for the analysis of the international trade network of aquatic products. Aquacult Int 28:1195-1211. https://doi.org/10.1007/s10499020-00520-5. 